\title{
Application Development of Virtual Reality Based on Smart Phone and VR Glasses
}

\section{Cao Yanzhen}

Zhengzhou University of industrial technology, Zhengzhou, Henan, 451100

Keywords: Unity 3D; virtual reality; VR glasses; cell phone sensor

\begin{abstract}
With the development of modern science and technology, many APP and VR can effectively combine and provide a series of virtual reality educational resources of education and teaching related resources, APP, so that you can feel the experience and experience the learning process. This paper focuses on the development process of a simple virtual reality application, mainly introducing the realization of the stereoscopic visual effect of VR glasses, the built-in sensor of the smart phone, and the setting of the scene. These are not available on the traditional media equipment, based on the virtual reality application development of VR glasses and smart phones, using the Unity $3 \mathrm{D}$ engine as the virtual reality software development environment, the basic three-dimensional visualization principle, combined with the key technology of VR and the development of virtual reality, and based on the mobile tracking gyroscope. Mobile accelerometer and virtual interaction mode interconnect, thus forming a set of VR virtual system. The development of the system only requires VR glasses, smart phones and Unity 3D engines to develop a virtual reality application, and enjoy the wonderful feeling of being in the world. The cost of the hardware is relatively low, which can provide a perfect virtual reality test for the user.
\end{abstract}

\section{Introduction}

At present, the virtual somatosensory game experience is spread all over the country and belongs to one of the most widely used applications of VR. Many people do not understand the virtual reality technology, and the knowledge is limited to VR glasses. In fact, the development of science and technology began with people's dissatisfaction with existing technologies. People were tired of the quality of 2D game images. They began to pursue high-quality sensory experiences, resulting in 3D images. Based on virtual reality application development of VR glasses and smart phones, Unity 3D engine is used as a virtual reality software development environment, basic 3D visualization principles, combined with key technologies of VR, realization of virtual reality application development, tracking of gyro and mobile phone acceleration based on mobile phones Meter, as well as the interconnection of virtual interactive mode, thus forming a set of VR virtual systems. The development of the system requires only some VR glasses and smartphones. These hardware inputs are relatively low cost, and this alone can provide users with a perfect virtual reality experience.

Many people believe that Virtual Reality creates a three-dimensional virtual world that reflects the changes and interactions of physical objects through simulation, and provides a three-dimensional boundary so that users can observe and interact with the virtual world through auxiliary perception equations. Such as helmet display and data gloves, so that users can directly participate in and explore imitation. The VR support technology is generally considered to be computer technology, analog technology, microelectronic technology, computer graphics, stereoscopic display technology, computer vision, multimedia technology, information technology, sensing and measurement technology, software engineering, speech recognition and synthesis technology, Man-machine interface technology and many other high-tech.

\section{Stereoscopic Visual Effects of VR Glasses}

Based on the development of virtual reality applications for smart phones and VR glasses, 
Cardboard, which is a box-type VR glasses, can be selected. These existing interactive technologies each have their own advantages and disadvantages and hardware characteristics. The interaction mode of this type of VR glasses is mainly "Timed aiming point" is what we usually call "gazing." Similar to the common children's shooting game, when the center of shooting and the gaze center overlap, the user knows where he is aiming, and can design a style for the center of mind, for example, the center is indicated by a cross plus sign or red dot, and the surrounding It is a semi-transparent ring. If the user's head is unstable and causes the user to operate erroneously, the user may be allowed to re-operate for a certain period of time to ensure the correctness of the selection. When the user enters an application or opens the game for use, in which the VR glasses need to be given to the user on the other side, when the other user's vision deviates from the main interface, the turn-by-turn indication of the arrow icon is required. For example, when the user's line of sight deviates from the positive direction by $50^{\circ}$, an arrow appears, and the head implies that the arrow can set a fade-out effect of 0.8 seconds.

Adding two virtual cameras in the Unity 3D engine, just like the human eyes in real life, to build a stereoscopic visual effect, build a simple virtual environment by adding two cubes to the scene. At $2,0,3)$ and $(2,0,3)$, paste different textures. Left $(-2,0,3)$ is given a "left" texture, right $(2,0,3)$ is given a "right" texture. Next, in the Scene in the Unity 3D engine, add two virtual cameras, one is Camera1 and the other is Camera2. Then you can virtually imitate the relationship between human eyes and the objects being viewed, and virtual cameras in the Scene and objects. The relative relationship. In order to increase the sense of realism, the two virtual cameras should set the corresponding left and right eye coordinates according to the distance between the two eyes of the real person.

VR forms a closed virtual reality experience space for the user through a specific helmet, allowing the user to feel the virtual scene in the panorama from the image of the helmet. The VR image is mainly user-oriented. As the user's body or way of thinking changes, it will open different pictures and content. Currently, Sony, HTC, and Samsung have all launched virtual reality experience devices, and nearly 90 companies in China are engaged in device development in VR. However, the lack of content and homogeneity in the experience as well as the feeling of dizziness and vomiting is also a huge disadvantage in the development of virtual reality today. Unlike 3D, 3D tourists do not directly open $3 \mathrm{D}$ paths, but use the motion of the images in the images to adapt to the space and scenes, thereby producing effects.

Virtual Reality Technology (VR) is an interactive immersive virtual three-dimensional space that provides users with computers. Its characteristics are mainly perception, interaction and immersion. Multi-sensory VR refers to visual perception, touch, motion perception, taste, and smell. Interactivity, that is, when users use VR technology, they can interact with them and get the same feelings as in real life, such as dismantling machines, changing equipment, and so on. The biggest advantage of virtual reality technology is to allow users to immerse themselves, that is, to provide users with a real environment, so that users feel like they are in reality.

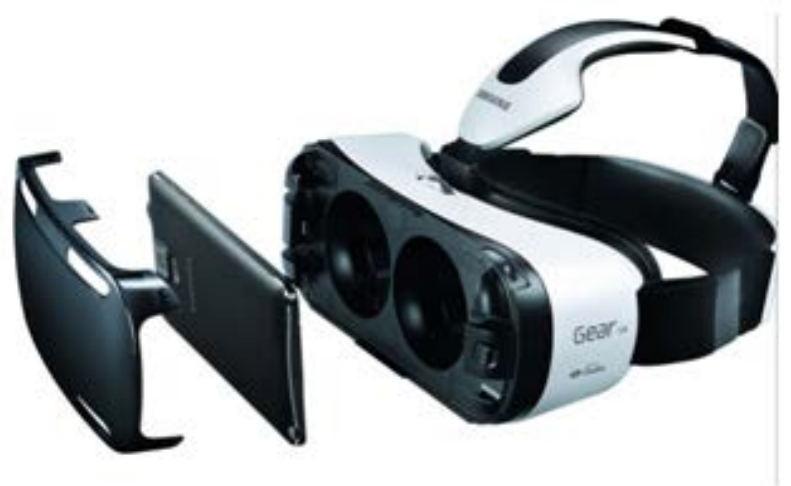

Figure 1 Mainstream mobile phone VR glasses 


\section{Introduction to Smart Phone Sensors}

The mobile phone gyroscope is also called the angular velocity sensor of the mobile phone. The function of the gyroscope is to detect the angular velocity of the mobile phone. That is to say, the measured physical quantity is the tilt and the angular velocity. This is different from the acceleration sensor. Only the acceleration sensor and not The angular velocity sensor has no way to measure some three-dimensional motion, it can only detect some axial linear behavior. However, the mobile gyroscope can well measure the rotation and deflection motion. The gyroscope chip lacks rotating parts and only has a vibrating piece, which can accurately determine and analyze the user's actual movement and convert the signal format of the mobile gyroscope. So that the smart phone can recognize, and then according to this action, the corresponding mobile phone operation can be carried out. Therefore, the mobile gyroscope can control the game through motion sensing. This important function provides a more realistic and somatosensory effect to the development experience of virtual reality.

Currently, smart phones on the market generally have gyro sensors built in. The angular speed sensors in smart phones are generally three-axis. It can be used to detect the head posture when the user experiences. When the smart phone rotates, the Coriolis force can calculate the rotational angular velocity along the axis direction under the effect of the three-dimensional deflection of the vibration component. According to the angular speed of the mobile gyro, the rotation angle can be directly obtained, and the posture angle of the smart phone can be calculated. Taking the VR classroom as an example, the school bus evacuation drill (student's perspective), when the students are wearing VR glasses and shaking the smart phone around looking around, the gyroscope can do a good measurement of the rotation, deflection movements, through The mobile phone window feeds back to the student so that the student can perform the next operation on the mobile phone. The virtual reality application developer can feedback data according to the corresponding actions of the mobile gyroscope, so that the VR application can be accurately operated and experienced.

The mobile phone gravity sensor can sense some electrical signals with acceleration or deceleration displacement, provided that these displacements are converted by G-sensor. Through computer analysis and calculation, programmers can program functions, such as QQ movement, the user takes the mobile phone. The body can calculate the number of steps for the user. In addition, the screen of the mobile phone may be sensed by a mobile phone gravity sensor. For example, when the user watches a video, the mobile phone is just started in a vertical state. When the user tilts the mobile phone to a certain degree, the screen is automatically converted to a horizontal state. The mobile phone gravity sensor can also sense the acceleration force. When the mobile phone falls, the mobile phone sensor senses the acceleration force and protects the mobile phone. The accelerometer of the mobile phone is whether the sensing mobile phone is stationary or accelerating. The acceleration sensor can use the smartphone to shake and shake to increase the realism of the mobile game. It can also sense whether the mobile phone falls down. The working principle of the mobile acceleration sensor is that the sensitive component converts the acceleration signal of the measuring point to the corresponding electrical signal and enters. The front-end circuit is set up, and the signal-to-noise ratio of the signal is improved by the signal therapy circuit, and then digital signals are obtained by analog-to-digital conversion. Finally, the digital signal is sent to the computer and the data is stored and displayed by the computer.

The acceleration sensor (G-sensor) measures the acceleration. The gravity acceleration sensor only measures the gravity. When the mobile phone is stationary, the readings of the two sensors should be the same, but during the movement, the acceleration sensor shows the acceleration of acceleration and motion. The acceleration is generated while the gravity sensor value shows the acceleration of gravity. A low-pass filter can be used to filter the acceleration sensor data to separate the acceleration of gravity. The force of the mobile phone in different physical directions can be sensed using an acceleration sensor. Acceleration sensors can sense the acceleration in three directions of $\mathrm{x}, \mathrm{y}$, and $\mathrm{z}$. Therefore, the acceleration sensor (G-sensor) can measure the acceleration in the three-dimensional space such as the horizontal direction and the vertical direction, not only the vertical direction considered by the user. With the aid of a gyroscope, the accelerometer can 
measure the gravity of the object more accurately.

\section{Virtual Reality Application Development}

Create a new Unity scene, create a new object (using Shift-Command-N), inspector panel (instpector), contains two parts, a "GameObject" and a "Transform". When you add a new object, the transformation component is automatically generated, so all objects have a transformation component. In Unity, the transformation component provides a unique function for all objects. It defines the position, rotation, and scaling of game objects in the scene view. Transform can also use the Parenting feature, that is, when an object is the parent object of another object, the rotation of this object will affect all child objects. You can create a parent by dragging any object to another object in the hierarchy view. The two objects will become parent-child relationships, also known as an object contained by another object. The script can access the corresponding local and global coordinates of the parent-child relationship.

When you add a script to the created game object, the saved script will become a component. When you write the script, you can access any member through the member list of the game object class. If the component the user wants to access is not included in the game as a member of the object, you need to use the game Object.GetComponent() component, which is the function module of the game object. All game objects contain a (Transform) component. If there is a default, the game object will not have a position. Create a new object, add a rigid body to the object, and then select it through the menu Rigidbody. The rigid body can work with any collision object. The collision device allows the rigid body to collide and interact with other colliders. The rigid body can be controlled by the Ageia PhysX physics engine. . To create a set of moving particle systems, use Particle Animator, Particle Renderer, and Particle Emitter. When you add a component to an object, it has different tunable values or attributes, and it can also be adjusted in the game through a JavaScript script.

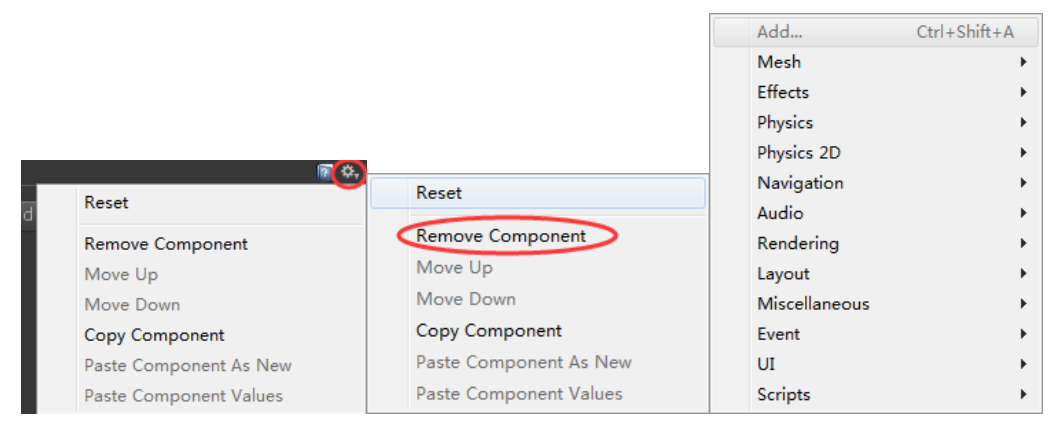

Figure 2 Adding Unity Components

In Unity 3D, there are three types of lights: point light, direction light, and projection light. Lights are widely used in scenes. The shape and appearance of the scene are determined by the grid and texture. The color and atmosphere of the scene are determined by Light-dependent. Real-time lighting can give users a good experience, but due to the limited use of smartphones in the development of virtual reality applications based on smart phones and VR glasses, too much real-time lighting will lead to some increase in the number of renderings, and finally reflected in the screen. Severe jitter, dizzy Caton phenomenon. In order to solve the above problem, only one real-time light is selected in the game, and the other is used as baking light. The light probe can produce a simulated light, but this light is a static light that does not render repeatedly when the game starts rendering. The advantage of this is that the rendering efficiency is greatly improved, only need to render once, without the need to repeat the rendering. In the setup of the Unity scene, the camera is indispensable. In the construction of the scene, you can create multiple cameras. Through the setting and operation of the camera in the game, you can add a sense of reality and uniqueness to the game. These cameras can be set. Add mystery to the game for any rendering position and sequence.

Unity 3D has a wide range of applications for three-dimensional vectors. For example, the speed 
and acceleration of an object, the direction of camera observation, and the force applied to a rigid body are all vectors. The operations include multiplication, point multiplication, and cross product. In the case of dot multiplication, if both vectors $a$, $b$ are unit vectors, then a.b is equal to the length of the projection of vector $b$ in the direction of vector a. E.g:

$$
\mathrm{a} \cdot \mathrm{b}=|\mathrm{a}||\mathrm{b}| \cdot \cos \theta, \cos \theta=(\mathrm{a} \bullet \mathrm{b}) /|\mathrm{a}||\mathrm{b}|, \theta=(\operatorname{Acos}(\cos \theta) * 180) / \pi
$$

For example, the value of a multiplication of two vectors is a three-level determinant. The result is a normal vector that is perpendicular to two vectors.

Based on the virtual reality system of smart phones and VR glasses, smart phones in box-type VR glasses appear as computing tools and display screens. The smart phones have sensors built therein and can sense changes in the positions of human heads through mobile phone gyros in order to realize The roaming of the virtual environment requires the control of the displacement of the virtual camera. Therefore, it is necessary to add a mobile phone and a mobile phone program in the virtual glasses for communication. The virtual camera is the basis of the displacement of the virtual scene, and its displacement can be divided into four directions (front, back, left, and right). The displacement control of the virtual camera of the mobile phone is essentially based on four front, rear, left, and right directions. Directional displacement creates a virtual camera and a smartphone's data map.

The triaxial accelerometer can measure the components in $\mathrm{x}, \mathrm{y}, \mathrm{z}$ directions by acceleration in the mobile phone. Untiy 3d supports this function. First look at the change of the $\mathrm{x}$ axis. Under the condition of the smartphone screen facing up, the mobile phone is above the phone. Toward the right, keep the phone horizontally tapped. If 90 degrees rotates to the right, then the gravity component will increase by 1; if 90 degrees rotate to the left, the gravity component will decrease by 1 . Look at the change of Y-axis. Under the condition that the smartphone screen faces upwards, the top of the mobile phone faces the right side to keep the horizontal tap of the mobile phone. If the outside of the mobile phone rotates 90 degrees outwards, that means the mobile phone screen faces outward, the gravity component will increase by 1; if 90 Rotation within the degree, that is, the phone screen inward, the gravity component is reduced by 1 . Looking at the changes in the Z-axis, the gravity component will increase by 1 if the smartphone screen faces downward; if the smartphone screen faces upward, the gravity component will decrease by 1 . It follows that the placement of the handset in different directions can be represented by components of the $\mathrm{X}, \mathrm{Y}$, and $\mathrm{Z}$ axes, and the component of the $\mathrm{Y}$ axis is represented by Input. acceleration.y, when $-1<$ Input.acceleration. $<<0$, Indicates that the phone screen is facing inwards. When $0<$ Input. acceleration. $y<1$, the mobile phone screen is facing outward.

After the user experiences, the user selects elements on the $\mathrm{X}$-axis and Y-axis as control signals. If the user is holding the mobile phone horizontally and the screen is vertically upwards, the mobile smart phone is tilted to the left and right. If you start to move the mobile phone to the left, the mobile phone sends a left signal to the mobile phone in the VR glasses case to move the virtual camera to the left. If the phone is tilted to the right, the phone sends a signal to the phone in the VR glasses case to move the virtual camera to the right. In this case, it is necessary to define a threshold, which cannot be too large or too small. The empirical value is generally set at 0.35 . If the threshold is too small, a slight tilt of the mobile phone will send a mobile signal, which will have a sensitive response. If the threshold is too large, the mobile phone will have a mobile signal with excessive tilt, and the response will be too slow. If the phone is tilted inwards, the phone sends a signal to the phone in the virtual glasses case and the virtual camera moves backward. Realize the communication between two mobile phones, here is the way of wireless network connection, Unity $3 \mathrm{D}$ package developer uses the network interconnection type, adopts C/S framework, uses the network to set the remote control smartphone into The client can use the smart phone in the VR glasses case as a server, waiting for other clients to connect. With the addition of head tracking, binocular stereo vision, and virtual camera somatosensory control, after installing smartphones and VR virtual reality glasses, a virtual reality application based on smart phones and VR glasses was successfully developed. This virtual reality system has the characteristics of simple structure, low 
price and strong versatility.

\section{Conclusion}

With the rapid development of virtual reality technology, the low cost of equipment has greatly reduced the threshold for the development of virtual reality systems. In the past, when many users used VR glasses, like watching 3D movies, virtual reality glasses were not fully studied in the direction of experience. Nowadays, with the development of science and technology, many APPs and VRs are effectively combined. For example, VR VR Classroom is a virtual reality education resource app that uses high-tech virtual reality technology to provide educational and teaching-related resources. It is a domestic focus on VR. The sharing app for educational resources allows you to experience the learning process on the ground. These are not possible on traditional media devices. For example: emergency education escape skills, help Komatsu, I will recognize time, interesting knowledge alone, etc. For these resources, we also only need VR glasses and smart phones to enjoy the immersive feeling.

\section{References}

[1] GUO Xiaoming. Virtual Reality System Based on VR Glasses and Smart Phone[J]. Master thesis of Xidian University, 2015.

[2] Liang Yirong. The application of virtual reality in digital entertainment games [J]. Brand Monthly, 2014, 08.

[3] Wu Yafeng, Yu Fuxing. Detailed and typical examples of Unity 3D game development techniques [M]. Beijing: People's Posts and Telecommunications Press, 2012.

[4] Lei Qiaoming. Research on the application of VR technology in English teaching [J]. Chinese Education Technology Equipment, 2016(12):131-132.

[5] Xuan Yusong. Unity 3D Game Development [M]. Beijing: People's Posts and Telecommunications Press, 2012.

[6] Yin Yue. Research and Development of Smartphone Baseband Circuit Based on MSM7227 Processor[J]. Donghua University Master Thesis, 2012. 